\title{
Technologies for Making Reliable Decisions on a Variety of Effective Factors using Fuzzy Logic
}

\author{
Yousef Ibrahim Daradkeh ${ }^{1}$ \\ Department of Computer Engineering and Networks \\ College of Engineering at Wadi Addawasir, 11991 \\ Prince Sattam Bin Abdulaziz University, KSA
}

\author{
Irina Tvoroshenko ${ }^{2}$ \\ Department of Informatics \\ Kharkiv National University of Radio Electronics \\ Kharkiv, Ukraine
}

\begin{abstract}
Problem of choosing methods has been presented for increasing the reliability of solutions while reducing the time resource for researching the state space of a particular object. Effective factors that can significantly affect the attainability of decision-making goals in a fuzzy process development environment are identified. Presented the selected factors in a classic form in natural language, which significantly increases the confidence in the decisions made by determining the maximum, minimum values of membership functions and the set of criteria by using manifest conflict situations when deciding. Information technologies and approaches for identifying the completeness of decision-making goals on a variety of effective factors are proposed. The effectiveness of the proposed solutions using the fuzzy inference procedure and the Zade-Mamdani's approach is estimated. Software testing carried of the described methods for evaluating and deciding on a variety of factors out using objectoriented programming. Experimental testing of realized ideas has confirmed an increase in the reliability of making aim management decisions in various applied subject areas.
\end{abstract}

Keywords-Attainability; completeness of decision-making; conflict; factor; membership function; state space

\section{INTRODUCTION}

Effective solutions in complex control systems and data processing of artificial intelligence systems of subject areas require the operational consideration of many conflicting factors: the essentially fuzzy nature of the interacting dynamic processes and the spaces of their states, the complex parallelsequential interaction of processes in the face of uncertainty [1], the significant complexity of the tasks, the significant share of human a factor that largely determines the quality and level of modern solutions, and others.

Implementing control, data processing, and decisionmaking processes in the computing intelligence systems of distributed complexes operating in a fuzzy environment [2], requires solving several problems [3].

These include analysis [4], identification [5], localization, and elimination of conflict situations during the interaction of processes in a fuzzy state space [6].

It is urgent to propose and develop a systematic approach to solving this problem in the form of constructing new mathematical fuzzy models, an adequate set of formal criteria, modern approaches, information technologies, effective methods and tools for solving a wide class of theoretical and applied problems.

\section{REVIEW OF THE LiterATURE}

The problem of goal attainability analysis is one of the main tasks of fuzzy process analysis [5]. In the process of creating computing intelligence systems, the developer has many goals, the quality of implementation, of which determines the degree of development efficiency. The goal will be realized in an environment of interacting processes. With inadequate and/or incorrect presentation and interaction, may not realize some goals. This requires a set of actions to ensure the goals of decisions [7].

This paper discusses the question of analyzing the attainability of the goals of decisions that are formed because of fuzzy processes and can be reflected in a variety of relations "condition-action".

It greatly complicates the task [1] of analyzing fuzzy processes is greatly because of the influence of fuzzy properties on by using develop processes in the structures of knowledge bases of intelligent systems [3] and decisionmaking based on them [5]. Existing approaches, given the complexity [6] and limited capabilities [7], do not allow us to get effective solutions. This requires using create new approaches and solutions-oriented to solving applied problems.

Qualitative accounting of many important factors [8] significantly affects the effectiveness of systems in practical implementations.

The primary problems of making reliable decisions [9] include ensuring: the attainability of decision-making goals, the consistency of decision-making goals, the completeness of decision-making, using conflict situations, using excess and useless loops, the quality of optimizing the choice of process development alternatives, the quality of optimizing the laws of learning and setting up fuzzy models and their reflection on real objects.

There is a significant amount of scientific research [10], models [11], methods [12] and software products [13] that, with varying degrees of effectiveness, solve some aspects of creating systems [14] and approaches based on computational intelligence [15].

The problems of comprehensively providing an actual reflection of the interaction of certain dynamic fuzzy processes, minimizing resources and ensuring an adequate choice of many alternatives for the possible development of fuzzy processes on 
a variety of specific constraints and assumptions are very important.

However, they do not have an analytical interpretation, which has found effective practical application in solving complex problems [16-18] requiring individual or collective decisions.

Such problems have a highly specialized character [19] and are solved on a limited set of subject areas.

The result of the analysis of modern scientific and practical literature [4-7, 15-22] confirmed that research in this area is not efficient enough; there is no clear interpretation and visualization of the interactive features of specific dynamic fuzzy processes and complex systems.

\section{PRoBlem StATEMENT}

There are many interacting fuzzy processes $\left\{\tilde{P_{i}}\right\}, \mathrm{i} \in \mathrm{I}$. The state space of interacting processes is defined on set $\left\{\tilde{A}_{i}\right\}$, $i \in I$. The initial state of the development of processes is defined by the space of initial states $\left\{\tilde{A}_{o i}\right\} \subset\left\{\tilde{A_{i}}\right\}$. The final state of development of processes is defined by the space of final states $\left\{\tilde{A_{k i}}\right\} \subset\left\{\tilde{A_{i}}\right\}$, which defines the set of realized goals $\left\{C_{r}\right\}, r \in R$.

If the set of processes $\left\{\tilde{P}_{i}\right\}, i \in I,\left\{\tilde{P}_{i}\right\} \neq \varnothing$ is defined, the space of initial states $\left\{\tilde{A}_{o i}\right\},\left\{\tilde{A}_{o i}\right\} \neq \varnothing$, then the attainability of the goals of the decisions made is realized if a subset of $\left\{\tilde{A_{k i}}\right\} \subset\left\{\tilde{A_{i}}\right\}$ is reachable, and it is true:

$\forall \tilde{P}_{i} \in\left\{\tilde{P_{i}}\right\}, \forall \tilde{A}_{o i} \in\left\{\tilde{A_{o i}}\right\} \mid\left\{\tilde{A}_{k i}\right\} \neq \varnothing$

Justice (1) determines the truth $D s_{j}=$ true, $j \in J$.

Based on the above interpretation of the space of final states $\left\{\tilde{A_{k i}}\right\} \subset\left\{\tilde{A_{i}}\right\}$, as the set of realized goals $\left\{C_{r}\right\}, r \in R$, the necessity and sufficiency of (1) to ensure $D s_{j}=$ true, $j \in J$ is obvious.

The solution to the problem of analyzing the attainability of decision-making goals includes the study of the state space of interacting fuzzy processes and the manifestation of the validity of conditions (1). All developed provisions should be focused on modern information technologies [9-15].
The aim of this work is to increase the reliability of decisions while reducing the time to analyze the state space of an object by developing information technologies and approaches that allow us to assess the state of many factors and highlight the most important for making effective decisions.

\section{MATERIALS AND METHODS}

Some effective factors will be defined that can significantly affect the attainability of decision-making goals in a fuzzy environment [23] for the development of processes [16].

A subset of the goals of decisions made $\left\{\tilde{D_{s_{j}}}\right\}$, for a given space of initial states $\left\{\tilde{A_{o i}}\right\}$, is unattainable if at least one of the following situations is true:

Situation 1.

$\exists \tilde{P_{i}} \in\left\{\tilde{P_{i}}\right\} \mid \mu_{P_{i}}\left(k_{0}\right)<\mu_{P_{i}}\left(k_{0}\right)^{*}$

Where $\mu_{\tilde{P}_{i}}\left(k_{0}\right)^{*}$ is the minimum permissible value of the membership function for $\tilde{P_{i}}$ processes; $k_{0}$ - the value of variable $k$, which determines the specific value of function $\mu_{\sim}(k)$.

Situation 2. There is at least one process $\tilde{P}_{i} \in\left\{\tilde{P}_{i}\right\}$, which cannot be performed and is fair for it

$\exists \tilde{P_{i}} \in\left\{\tilde{P_{i}}\right\} \mid\left\{\tilde{D_{s_{j}}}\right\}=$ false, $\left\{\tilde{D_{s_{j}}}\right\} \neq \varnothing$

Situation 3. There is a state that does not stop the mutual expectation of two or more processes (tasks).

The achieved processes $\left\{\begin{array}{c}\sim \\ P_{i}^{\prime}\end{array}\right\}$ are a subset of processes $\left\{\tilde{P_{i}^{\prime}}\right\} \subset\left\{\tilde{P_{i}}\right\},\left\{\tilde{P_{i}^{\prime}}\right\} \neq \varnothing$ for which none of the conditions and (3) is satisfied.

The analysis of characteristic situations makes it possible to determine the following:

- Situation 1 determines that in order to solve the problem of attainability of goals (1), it is necessary to modify the values of $\mu_{P_{i}}\left(k_{0}\right)$ in (2) so that

$\forall \tilde{P_{i}} \in\left\{\tilde{P_{i}}\right\} \mid \mu_{P_{i}}\left(k_{0}\right) \geq \mu_{\tilde{P}_{i}}\left(k_{0}\right)^{*}$ 
To fulfill (4) in real systems, some additional resources are attracted [17], the nature, volume, and labor of which are determined by a specific subject area;

- Situations 2 and 3 reflect the fact of logical inadequacy of the goals of decision-making processes in the subject area [9], which necessitates the modification of the laws of interaction of fuzzy processes.

The conflict situation in the interaction of fuzzy processes is defined as the presence of at least one of the situations:

- Two or more processes compete for data that is obtained as a result of previous actions;

- Two or more processes compete for resources that ensure the following processes.

The presence of conflicts in the interaction of processes in complex objects leads to a significant decrease in the efficiency of functioning.

If the set of processes $\left\{\tilde{P}_{i}\right\}, i \in I,\left\{\tilde{P}_{i}\right\} \neq \varnothing$ is defined, the space of initial states $\left\{\tilde{A}_{o i}\right\},\left\{\tilde{A}_{o i}\right\} \neq \varnothing$, the space of current states $\left\{\tilde{A}_{i}\right\},\left\{\tilde{A}_{i}\right\} \neq \varnothing$, the space of final states $\left\{\tilde{A_{k i}}\right\},\left\{\tilde{A}_{k i}\right\} \neq \varnothing$, then there are no conflict situations if there is no competition for resources:

$$
\begin{aligned}
& \forall \tilde{P_{i}} \in\left\{\tilde{P_{i}}\right\}, \forall \tilde{A}_{o i} \in\left\{\tilde{A}_{o i}\right\}, \forall \tilde{A}_{i} \in\left\{\tilde{A_{i}}\right\}, \\
& \forall \tilde{A}_{k i} \in\left\{\tilde{A_{k i}}\right\} \mid \operatorname{Conf}_{k}=\varnothing
\end{aligned}
$$

subject to the fulfillment of (4).

Solving the problem of manifestation of conflict situations requires complex studies of the structure of the model in the space of model states and processes.

\section{EXPERIMENTS}

The solution of the problem of increasing the reliability of decisions [19] while reducing the time to analyze the state space of an object, taking into account (1) and the accepted interpretation (2)-(4), can be reflected in the form of technology for detecting the completeness of decision-making goals:

- The simulated processes (phenomena) occurring in the object of modeling and analysis are described by a set of events and conditions by which these events are determined, as well as causal relationships that are established on the set "condition-action";

- Set of "condition-action" define when control the sequence of occurrence, where is a specific space of potential states of the object of research. The state space is defined by many conditions. Conditions are formulated in the form of predicates and complex procedures;

- Conditions (predicates) may be fulfilled and not fulfilled. Only the fulfillment of the conditions provides the possibility of events. Conditions, the fulfillment of which is associated with the possibility of events, are "in-conditions";

- After the event has occurred, other conditions that are in-conditions and the consideration of events with a causal relationship will be satisfied, they are called “out-conditions".

Accept this interpretation of components of the model:

- The set of input conditions (antecedents) and the initial conditions (consequents) is described by the set of positions $\left\{\tilde{p}_{j}\right\}, j \in J$, and the set of events (actions) by the set of transitions $\left\{\tilde{t}_{i}\right\}, i \in I$;

- The sequence of events is displayed by performing transitions. The fulfillment of any condition is associated with the appearance of marker $\tilde{M}\left(\tilde{p}_{j}\right) \geq 1$ in the position corresponding to this condition. Transition triggering rules are a way of expressing causal relationships between conditions and events in a system. After all, in-conditions are fulfilled, events may occur;

- The moment of actual realization of events is unknown. Sometimes it is difficult to restore the complete chain of immediate causes and consequences that determine the fact and time of the duration of the event, the identification of the properties of clarity and so on;

- After the event has occurred, out-conditions are implemented. These out-conditions are in-conditions of other incident events that determine the desired result. The root causes may disappear or continue to function. This is because fuzzy models have the property of absorption and the generation of markers.

The dynamics of modeling on fuzzy models is associated with the mechanism for changing position markings and the rules for performing transitions in the model state space.

With a direct simulation procedure and fuzzy inference, transition $\tilde{t}_{i}$ is triggered for which

$\forall \tilde{p}_{j} \in\left\{\tilde{p}_{i}(\right.$ in $\left.)\right\} \mid \tilde{M}\left(\tilde{p}_{j}\right)>0$

With the reverse simulation procedure and fuzzy inference, transition $\tilde{t}_{i}$ is triggered for which

$\forall \tilde{p}_{j} \in\left\{\tilde{p}_{i}(\right.$ out $\left.)\right\} \mid \tilde{M}\left(\tilde{p}_{j}\right)>0$

The transition in the general case can occur after any finite period $\tau$ after the transition is triggered. Marking of all input and output positions associated with this transition is 
instantaneous, taking into account time delays $\tau$, provided that the corresponding predicate is true from $L$.

Result of performing transitions with a direct procedure, a given value of function $\tilde{\mathrm{F}}(\mathrm{f})$ and the accepted interpretation, only one marker is removed from each incoming position of this transition $\tilde{t}_{i}$ and exactly one marker is added to the markings of each initial position

$$
\begin{aligned}
& \left(\forall \tilde{p}_{j} \in\left\{\tilde{p}_{i}(\text { in })\right\} \mid \tilde{M}\left(\tilde{p}_{j}\right)-1\right) \text { and } \\
& \left(\forall \tilde{p}_{i} \in\left\{\tilde{p}_{i}(\text { out })\right\} \mid \tilde{M}\left(\tilde{p}_{i}\right)+1\right) .
\end{aligned}
$$

Result of performing transitions in the reverse procedure, given the value of function $\tilde{F}(f)$ and the accepted interpretation from each source position of this transition $\tilde{t}_{i}$, only one marker is removed and exactly one marker is added to the markings of each input position

$$
\begin{aligned}
& \left(\forall \tilde{p}_{j} \in\left\{\tilde{p}_{i}(\text { in })\right\} \mid \tilde{M}\left(\tilde{p}_{j}\right)+1\right) \text { and } \\
& \left(\forall \tilde{p}_{i} \in\left\{\tilde{p}_{i}(\text { out })\right\} \mid \tilde{M}\left(\tilde{p}_{i}\right)-1\right) .
\end{aligned}
$$

Preliminary actions are performed if:

- An acceptable value of fuzziness of processes $\left\{\tilde{P}_{i}\right\}$ is provided, which determines the permissibility of transitions $\exists \tilde{t_{i}} \in \tilde{T}$;

- The validity of some predicate $L\left\{x_{u}\right\}$ is satisfied, which displays the properties of sets of factors and functions $\tilde{F}(f)$, state spaces $\{\tilde{M}(f)\}$ of a fuzzy model;

- The truth of some procedure $\left\{\tilde{P} r_{j}\right\}$ reflects the properties of many factors and state spaces $\{\tilde{M}(f)\}$ of the fuzzy model.

Analysis of the completeness of decisions made using fuzzy models makes it possible, based on formal approaches, to determine the completeness properties of the goals of decisions made.

\section{RESUlT AND DisCUSSION}

Operators of fuzzy relations [1] using in the work make it possible to implement fuzzy inference procedures.

In a comparative analysis, the Zadeh-Mamdani's approach is more promising; he can be represented of rules

$$
\begin{aligned}
& \left\{\text { if } x_{i} \text { is } \mu(x) \text { then } y_{i} \text { is } \mu(y)\right\}, i \in I \\
& x_{i}=x^{\prime} \\
& y_{i}^{\prime}-\text { ? }
\end{aligned}
$$

Where $x^{\prime}$ is some received vector; $y_{i}^{\prime}$ - the desired solution for the case $i \in I$.

Procedure [8] on the basis of (5) implemented for $\forall x_{j}^{\prime}$, $x_{j}^{\prime} \in A$ with $A \neq \varnothing$ in the form of some composition of rules $|I|$, then for (10) have

$y^{\prime}=\wedge x^{\prime} \vee \mu(x, y)$

Where $\mu(x, y)$ - fuzzy relationship of antecedent and consequent with [1].

Defuzzification rules the based on the center of mass [1] get for (10) the desired approximate solution $y_{i}^{\prime}$. The value $y_{i}^{\prime}$ can be refined by adjusting membership functions [8] until achieve the required accuracy standard.

Particular attention is paid to maximizing the values of membership functions of the expected solutions in such a way

$$
\Delta \stackrel{F}{\longrightarrow} \text { extr }
$$

Where $\Delta-$ an error that may exceed some acceptable value; $F$ - restrictions determining the value of membership function.

Based on the developed methods for evaluating and making decisions on a variety of factors, fuzzy-type big data processing systems, presented in natural language in quantitative and qualitative form, have created a tool for constructing models.

Optimization criteria into account take: maximum clarity of components and processes, minimum time costs, maximum values of reliability indicators under given restrictions, and their derivatives and vector criteria.

Criteria and limitations are defined and can be specified by the features of functional tasks and the subject area.

It is advisable to focus on implementing modeling and analysis of processes on the capabilities of modern computer systems.

The structure of the developed tool provides:

- Tracking marker paths and calculating parameter values, conditions, predicates, and procedures;

- Assignment and processing of accessory functions for model components;

- Analysis of the attainability of the goals of decisions;

- Analysis of conflict situations;

- Analysis of alternatives to decisions (Fig. 1);

- Analysis of the completeness of decision-making goals;

- Analysis of the consistency of decision-making goals;

- Analysis of excess and useless loops;

- Modification of the model according to the results of analysis and modeling; 
- Determination of system time for solving applied problems.

Search for alternatives using the develop of dynamic fuzzy processes (Fig. 2) is carried out according to the criteria:

- Minimum time parameters;
- Maximum clarity values in decision-making procedures;

- Other parameters determined by the subject area (the criteria for choosing solutions based on these parameters may be: the sum of their values, average value, minimum, maximum values) (Fig. 3).

\section{Network Model Interpretation \\ TI Model example}
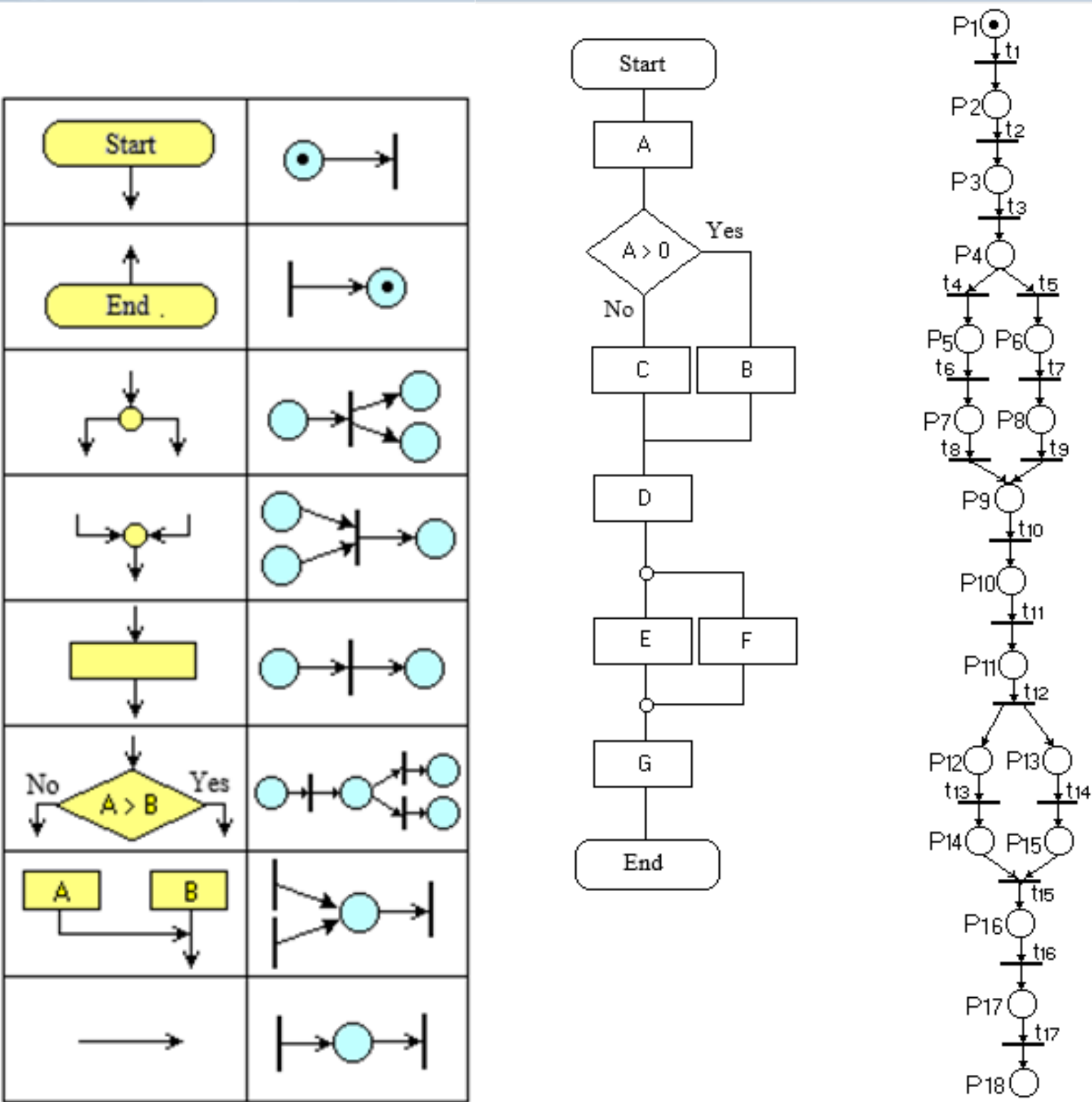

Fig. 1. Interpretation of Components of Network Models and an Example of Constructing a Fragment of the Model. 


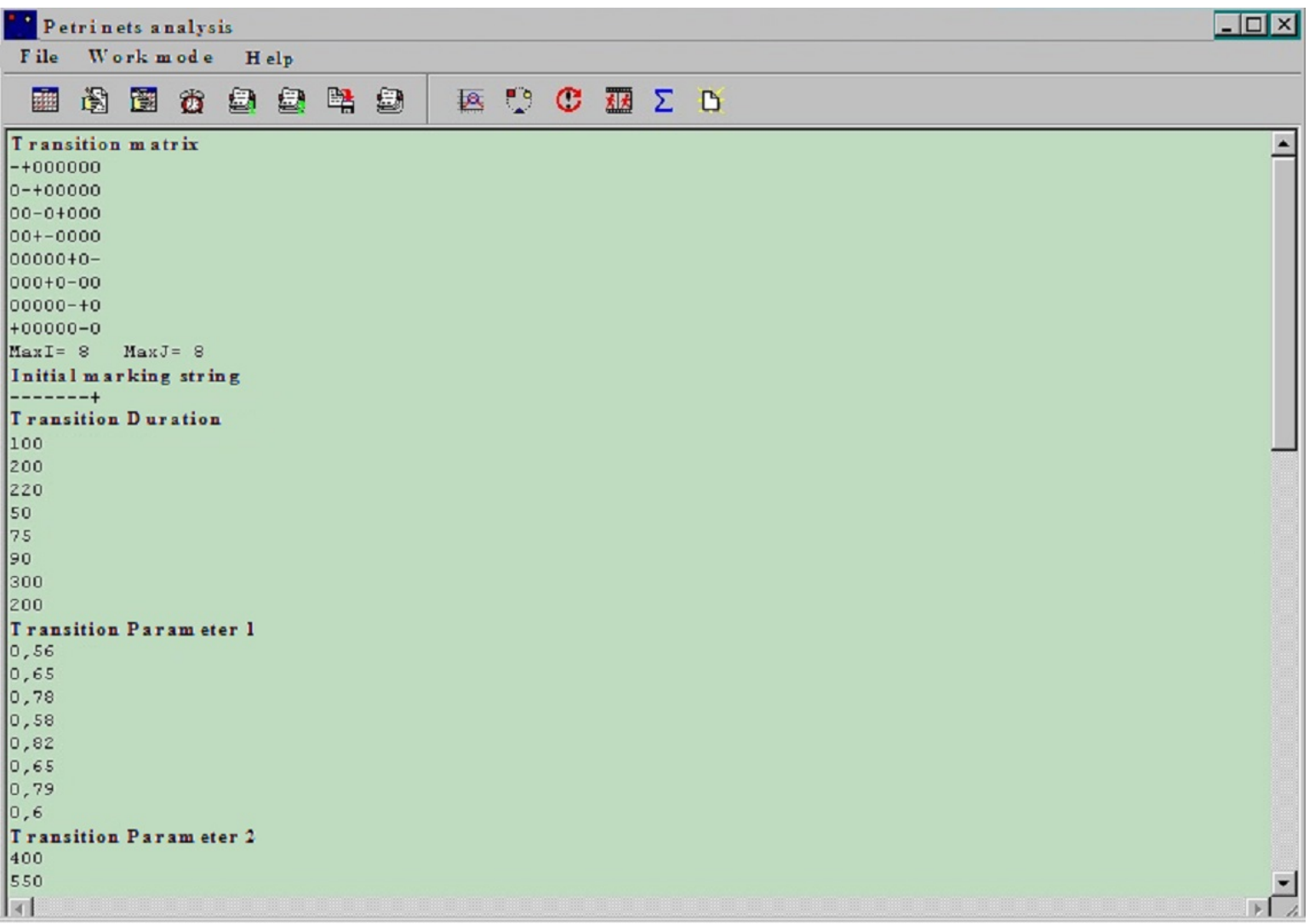

Fig. 2. The Result of Solving the Problem of Finding Alternatives.

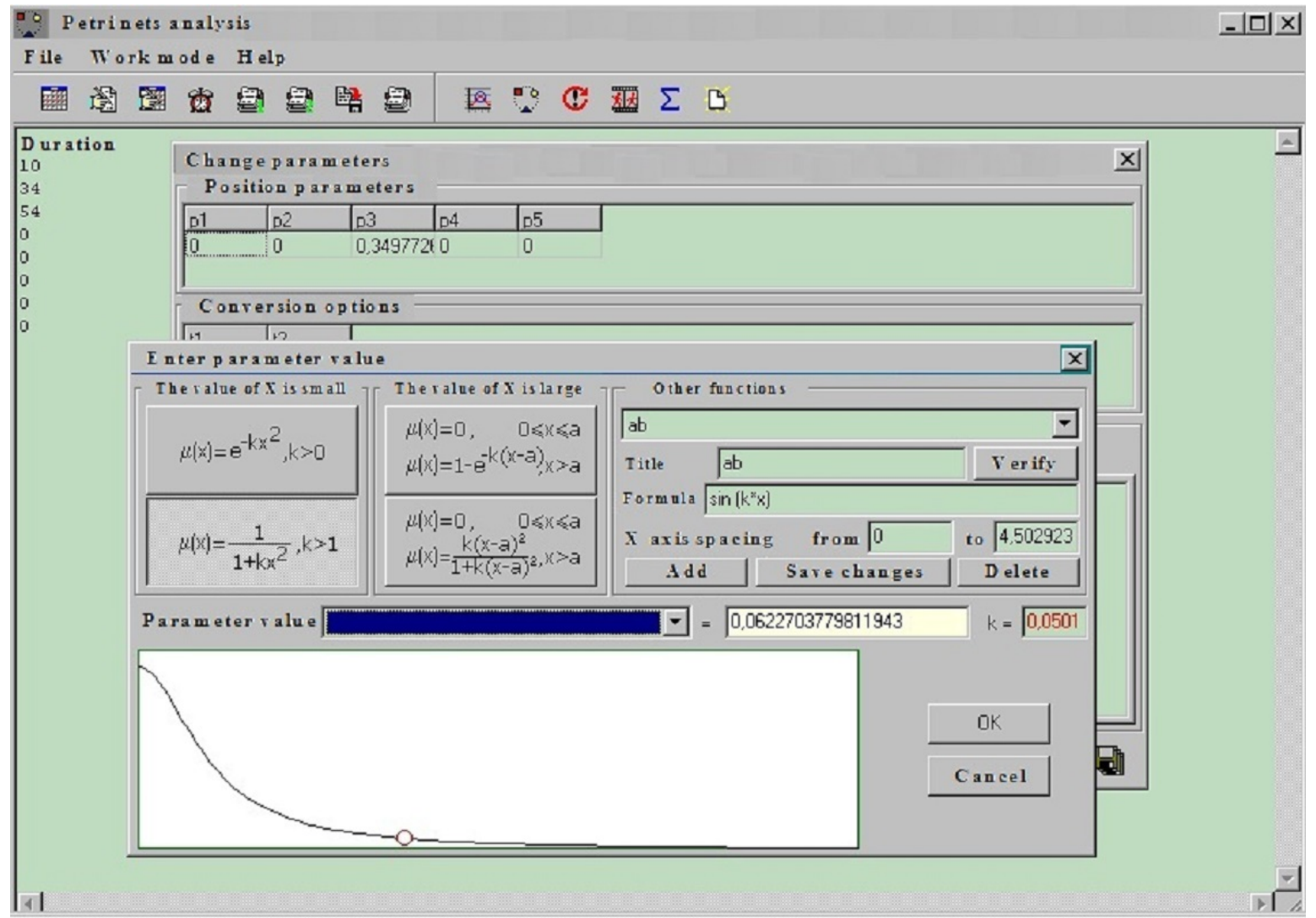

Fig. 3. The Mode of Forming Values of Membership Functions. 
For high-dimensional models, decomposition operations, which are often used for detailed research of models and the subject area. Applied problem solved the usage of the composition of the model on the fragment.

In various applied subject areas that work in optimal or extreme situations, it may be necessary to decide under conditions of fuzziness, uncertainty, the unreliability of time and resource constraints or assumptions.

The developed software application allows you to find a reliable solution based on these factors using the apparatus of computational intelligence, which reflects a complex of mathematical models, methods, and means of implementing dynamic interacting processes that operate in conditions of uncertainty.

The implementation of modern trends in the construction of control and data processing systems significantly expands the capabilities of managing facilities, reduces the cost of necessary resources. The traditional and intelligent components of the systems complement each other and function in constant interconnection. Fuzzy components of the description of management processes and decision-making are gaining importance.

\section{CONCLUSION}

Approaches have been developed to identify the completeness of decision-making goals on a variety of effective factors for complex objects operating in fuzzy state space.

The practical application of tools for modeling and analysis of fuzzy processes in solving a wide class of practical problems, identifying, localizing and eliminating the incompleteness and inconsistency of the knowledge bases of dynamic fuzzy systems is proposed. The presented development made it possible to increase the reliability of managerial decisions by $15 \%$ according to expert data while reducing by $28 \%$ the time resource for researching the state space of a particular object.

The convenience of the proposed approaches also lies because users of the tools can be specialists who do not have in-depth knowledge, skills in computer science and programming.

The software application is developed in an object-oriented programming environment. Testing and repeated testing of the proposed technologies and tools confirmed the high reliability of the adopted objective management decisions on the topic of research.

The proposed approaches determine the effectiveness and feasibility of using knowledge of oriented technologies for decision-making by means of fuzzy logic.

A promising area of new research is the improvement or further expansion of criteria, methods, algorithms and software on a variety of alternatives when making reliable decisions.

\section{REFERENCES}

[1] I. S. Tvoroshenko, and V. O. Gorokhovatsky, "Intelligent classification of biophysical system states using fuzzy interval logic,"
Telecommunications and Radio Engineering, Vol. 78, No. 14, pp. 13031315, 2019. https://doi.org/10.1615/TelecomRadEng.v78.i14.80

[2] M. Ayaz Ahmad, Irina Tvoroshenko, Jalal Hasan Baker, and Vyacheslav Lyashenko, "Computational Complexity of the Accessory Function Setting Mechanism in Fuzzy Intellectual Systems,” International Journal of Advanced Trends in Computer Science and Engineering, Vol. 8, No. 5, pp. 2370-2377, 2019. https://doi.org/10.30534/ijatcse/2019/77852019

[3] S. Ashraf, T. Mahmood, S. Abdullah, and Q. Khan, "Different approaches to multi-criteria group decision making problems for picture fuzzy environment," Bulletin of the Brazilian Mathematical Society, New Series, Vol. 50, No. 2, pp. 373-397, 2019. https://doi.org/10.1007/s00574-018-0103-y

[4] K. Zhang, J. Zhan, and W. Z. Wu, "Novel fuzzy rough set models and corresponding applications to multi-criteria decision-making," Fuzzy Sets and Systems, Vol. 383, pp. 92-126, 2020. https://doi.org/10.1016/j.fss.2019.06.019

[5] P. H. Thong, and S. Le, "Picture fuzzy clustering: a new computational intelligence method," Soft computing, Vol. 20, No. 9, pp. 3549-3562, 2016. https://doi.org/10.1007/s00500-015-1712-7

[6] T. Hanne, and R. Dornberger, "Computational intelligence," Computational Intelligence in Logistics and Supply Chain Management, pp. 13-41, 2017. https://doi.org/10.1007/978-3-319-40722-7_2

[7] V. A. Gorokhovatskyi, "Image classification methods in the space of descriptions in the form of a set of the key point descriptors," Telecommunications and Radio Engineering, Vol. 77, No. 9, pp. 787797, 2018. https://doi.org/10.1615/TelecomRadEng.v77.i9.40

[8] S. M. Aristova, Y. I. Daradkeh, and P. M. Korolev, "Processes in Experiences with Uncertainty: How to Approach,” Journal of Materials Science \& Nanotechnology, Vol. 5, No. 1, pp. 106-110, 2017. https://doi.org/10.15744/2348-9812.5.106

[9] Majed Kamel Al-Azzam, Malik Bader Alazzam, and Majida Khalid alManasra, "MHealth for decision making support: a case study of ehealth in the public sector," International Journal of Advanced Computer Science and Applications, Vol. 10, No. 5, pp. 381-387, 2019. https://doi.org/10.14569/IJACSA.2019.0100547

[10] Maruf Ahmed Tamal, Md Saiful Islam, Md Jisan Ahmmed, Md. Abdul Aziz, Pabel Miah, and Karim Mohammed Rezaul, "Heart disease prediction based on external factors: A machine learning approach," International Journal of Advanced Computer Science and Applications, Vol. 10, No. 12, pp. 446-451, 2019. https://doi.org/10.14569/IJACSA.2019.0101260

[11] W. Pedrycz, A. Sillitti, and G. Succi, "Computational intelligence: an introduction," Computational Intelligence and Quantitative Software Engineering, pp. 13-31, 2016.

[12] I. Perova, and I. Pliss, "Deep hybrid system of computational intelligence with architecture adaptation for medical fuzzy diagnostics," International Journal of Intelligent Systems and Applications, Vol. 9, No. 7, pp. 12-21, 2017. https://doi.org/10.5815/ijisa.2017.07.02

[13] B. Xing, and W. J. Gao, "Overview of computational intelligence," Artificial Intelligence: Concepts, Methodologies, Tools, and Applications, pp. 12-31, 2017. https://doi.org/10.4018/978-1-5225-17597.ch002

[14] Blessing Ekong, Idara Ifiok, Ifreke Udoeka, and James Anamfiok, "Integrated fuzzy based decision support system for the management of human disease," International Journal of Advanced Computer Science and Applications, Vol. 11, No. 2, pp. 268-274, 2020. https://doi.org/10.14569/IJACSA.2020.0110235

[15] I. J. Pérez, and et al., "On dynamic consensus processes in group decision making problems,” Information Sciences, Vol. 459, pp. 20-35, 2018. https://doi.org/10.1016/j.ins.2018.05.017

[16] D. Hamann, N. P. Walz, A. Fischer, M. Hanss, and P. Eberhard, "Fuzzy arithmetical stability analysis of uncertain machining systems," Mechanical Systems and Signal Processing, Vol. 98, pp. 534-547, 2018. https://doi.org/10.1016/j.ymssp.2017.05.012

[17] Sumaira Nazir, Nargis Fatima, and Suriayati Chuprat, "Situational factors for modern code review to support software engineers' sustainability,” International Journal of Advanced Computer Science and Applications, Vol. 11, No. 1, pp. 498-504, 2020. https://doi.org/10.14569/IJACSA.2020.0110161 
[18] January D. Febro, "Utilizing feature selection in identifying predicting factors of student retention," International Journal of Advanced Computer Science and Applications, Vol. 10, No. 9, pp. 269-274, 2019. https://doi.org/10.14569/IJACSA.2019.0100934

[19] P. Pourhejazy, J. Sarkis, and Q. Zhu, "A fuzzy-based decision aid method for product deletion of fast moving consumer goods," Expert Systems with Applications, Vol. 119, pp. 272-288, 2019. https://doi.org/10.1016/j.eswa.2018.11.001

[20] M. J. Knowling, J. T. White, and C. R. Moore, "Role of model parameterization in risk-based decision support: An empirical exploration,” Advances in water resources, Vol. 128, pp. 59-73, 2019. https://doi.org/10.1016/j.advwatres.2019.04.01

[21] J. J. Rainer, S. Cobos-Guzman, and R. Galán, "Decision making algorithm for an autonomous guide-robot using fuzzy logic," Journal of
Ambient Intelligence and Humanized Computing, Vol. 9, No. 4, pp. 1177-1189, 2018. https://doi.org/10.1007/s12652-017-0651-9

[22] Subashini Ganapathy, Zulkefli Mansor, and Kamsuriah Ahmad, "Investigating factors affecting knowledge management practices in public sectors," International Journal of Advanced Computer Science and Applications, Vol. 10, No. 11, pp. 205-212, 2019. https://doi.org/10.14569/IJACSA.2019.0101128

[23] I. S. Tvoroshenko, and V. O. Gorokhovatsky, "Modification of the branch and bound method to determine the extremes of membership functions in fuzzy intelligent systems," Telecommunications and Radio Engineering, Vol. 20, No. 78, pp. 1857-1868, 2019. https://doi.org/10.1615/TelecomRadEng.v78.i20.80. Available online: http://www.dl.begellhouse.com/ru/journals/0632a9d54950b268,55c1ca0 a73cc00be,339e67477ec4f851.html 